\title{
Unusual clinical manifestations of dengue infection in children in a tertiary care hospital in northeast Thailand
}

\author{
Tanaporn Duangmala, Pagakrong Lumbiganon, Pope Kosalaraksa \\ Department of Pediatrics, Faculty of Medicine, Srinagarind Hospital, Khon Kaen University, \\ Khon Kaen 40002, Thailand
}

\begin{abstract}
Background: Dengue virus infection has been a public health concern in Thailand. In the past decades, there has been recent interest concerning unusual clinical manifestations in both dengue fever (DF) and dengue hemorrhagic fever (DHF).

Objective: We described the unusual clinical manifestations and outcomes of children with dengue admitted to a tertiary care hospital in northeast Thailand.

Materials and Methods: A study was conducted on the 73 patients with serologically confirmed dengue infection admitted to Srinagarind Hospital, a tertiary care facility in northeast Thailand between January 2007 and August 2011.

Results: Of the 73 children examined, 42 (57\%) were boys and 31 were girls. Their age ranged from 8 months to 14 years (median 11 years). Nine patients developed neurological symptoms, 6 patients had altered consciousness, and 3 patients convulsion. Among 9 patients with neurological symptoms, 1 patient had acute kidney injury, 1 had hepatic failure, and 1 had kidney and liver involvement, mostly associated with fluid resuscitation or prolonged shock. Apart from neurological symptoms, one patient developed infection associated hemophagocytic syndrome and was treated with intravenous immunoglobulin. Two patients died from multiple organ failure, and 1 patient was brought back home in a moribund condition. The other patients recovered completely.

Conclusion: Altered consciousness was the most commonly observed unusual neurological manifestation. Patients who did not develop acute kidney injury or liver failure had mild clinical courses and recovered from neurological symptoms without sequelae. Acute kidney injury was associated with fluid overload and/or prolonged shock. Careful fluid management and close monitoring for complications resulted in favorable outcomes.
\end{abstract}

Keywords: Dengue fever, dengue hemorrhagic fever, dengue shock syndrome, dengue virus infection, unusual manifestation

Dengue virus infection has been a public health concern in Thailand and in other tropical countries worldwide. Infection may be asymptomatic or may present as an undifferentiated febrile illness or as dengue hemorrhagic fever (DHF) including dengue shock syndrome (DSS) [1]. DHF and DSS are classified into 3 phases: febrile, critical, and convalescent. The critical phase of DHF, i.e. the period of plasma leakage, begins during the transition from febrile to the afebrile phase. The plasma leakage may lead to hypovolemic shock (DSS). Patients with prolonged or uncorrected shock may develop a more complicated course with metabolic acidosis and

Correspondence to: Dr. Pagakrong Lumbiganon, Department of Pediatrics, Faculty of Medicine, Srinagarind Hospital, Khon Kaen University, Khon Kaen 40002, Thailand. E-mail: paglum@ kku.ac.th electrolyte imbalance, multiple organ failure, and severe bleeding from various organs. Prolonged shock can lead to hepatic and renal failure. Encephalopathy may occur in association with multiple organ failure, metabolic and electrolyte disturbances, or as an isolated syndrome.

There have been an increasing number of reports of atypical or unusual clinical manifestations such as encephalopathy, encephalitis, acute hepatitis, and acute kidney injury in both children and adults with dengue infection [2-10]. The World Health Organization Comprehensive Guidelines for Prevention and Control of Dengue and Dengue Haemorrhagic Fever 2011 [11] described expanded dengue syndromes as clinical features of symptomatic dengue infection. This included patients with severe organ involvement of liver, kidney, brain, or heart that are associated with dengue infection. These unusual manifestations may 
be associated with other co-infections, comorbidities, or complications of prolonged shock.

There are few reports of unusual manifestations in dengue infected Thai children. The incidence of neurological manifestations was reported to be 5.4\% in 1,493 serologically proven case of dengue infection in children in a tertiary care center in Bangkok from 1987 to 1998 [3]. High mortality rates of 64\% and $50 \%$ were reported in fulminant hepatic or renal failure in children $[9,10]$. This study aims to review unusual clinical manifestations and outcomes of children with dengue admitted to a tertiary care hospital in northeast Thailand with particular focus on the prevalence, clinical course and outcome.

\section{Materials and methods}

Medical records of all patients diagnosed with DF, DHF, and DSS admitted at Srinagarind Hospital, Khon Kaen University from January 2007 to August 2011were reviewed. Those aged below 15 years were included in this study. Patients with positive dengue NS1 Ag and/or dengue IgM using immunochromatography (Dengue NS1Ag Duo Rapid Test, SD Bioline, France) [12] were categorized as DF, DHF, and DSS according to WHO guidelines [11]. The presence of abnormal hemostasis such as positive tourniquet test and leakage of plasma was used to differentiate DHF from DF. The grading system of DHF includes Grade I — positive tourniquet test, Grade II—spontaneous bleeding, Grade III—hypotension, Grade IV - circulatory failure. Grades III and IV DHF are also referred to as Dengue Shock Syndrome (DSS).

Unusual manifestations and laboratory findings were correlated with the day or phase of illness in an attempt to define causes of the unusual manifestations. Unusual manifestations that occurred after the critical phase (fever subsided) or shock and receiving fluid resuscitation for more than 24 hours were defined as complications. Coinfection was defined by a positive culture or serology for an additional infectious agent. Fulminant hepatic failure was defined as biochemical evidence of acute liver injury (usually $<8$ weeks duration); no evidence of chronic liver disease; and coagulopathy. This was defined as a prothrombin time (PT) $>15 \mathrm{~s}$ or international normalized ratio (INR) $>1.5$ not corrected by vitamin $\mathrm{K}$ and in the presence of clinical hepatic encephalopathy, or a PT $>20$ s or INR $>2$ regardless of the presence of clinical hepatic encephalopathy [13]. Acute kidney injury was defined as an abrupt (within $48 \mathrm{~h}$ ) reduction in kidney function, absolute increase in serum creatinine of $\geq 0.3 \mathrm{mg} / \mathrm{dl}$, a percentage increase in serum creatinine of $\geq 50 \%$ (1.5-fold from baseline), or a reduction in urine output $(<0.5 \mathrm{ml} / \mathrm{kg}$ per hour for $>6$ hours) [14].

All quantitative data were expressed as mean \pm standard deviation or median where appropriate. This study was approved by the Ethics Committee for Human Research of Khon Kaen University

\section{Results}

During January 2007 to August 2011, 224 children ( $<15$ years old) were admitted to our hospital with a diagnosis of DF, DHF, and DSS. Dengue NS1Ag and dengue IgM were tested in 100 patients, 73 had positive results. Positive NS1Ag was found in 16 patients with median day of illness tested as 4 days (range 1-6 days). Positive dengue IgM was found in 40 patients with the median day of illness tested 5 days (range 3-14 days). Both positive tests were seen in 17 patients with the median day of illness tested 7 days (range $3-8$ days). Most patients with confirmed cases of dengue $(63 / 73,86.3 \%)$ were hospitalized between 2009 and 2011 when rapid diagnostic tests for dengue were available in our hospital. Demographic data of these 73 patients are shown in Table $\mathbf{1}$. The proportion of boys and girls was 42 (57\%) and 31 (43\%) and the median age of patients was 11 years (range, 8 months to 14 years). The median duration of illness before hospitalization was 4.4 days (range 1-9 days). Among 73 patients, 56 (77\%) were hospitalized in the febrile stage with median days of illness before admission of 4.0 days (range 1 to 9 days). Another 17 patients (23\%) were hospitalized at the time that fever had subsided. About one quarter (18/73, 25\%) were referred to our hospital from other hospitals where they had been treated for at least 24 hours.

According to the WHO criteria [1], 31 (43\%) patients had DF, 29 (39\%) DHF (grades I and II), and 13 (18\%) had DSS (DHF grade III and IV) (Table 1). Seventeen patients (23\%) had at least one underlying disease such as thalassemia (6 patients), well-controlled asthma (5 patients), allergic rhinitis (2 patients), and one each with thalassemia and asthma, G-6-PD deficiency, acute T-cell lymphoblastic leukemia in maintenance phase and ventricular septal defect of functional class I. 
Table 1. Data of 73 patients with DF, DHF and DSS

\begin{tabular}{lcccc}
\hline Parameter & $\begin{array}{c}\text { DF } \\
(\mathbf{n}=\mathbf{3 1})\end{array}$ & $\begin{array}{c}\text { DHF } \\
(\mathbf{n}=\mathbf{2 9})\end{array}$ & $\begin{array}{c}\text { DSS } \\
(\mathbf{n}=\mathbf{1 3})\end{array}$ & $\begin{array}{c}\text { Total } \\
(\mathbf{n}=\mathbf{7 3}) \mathbf{( \% )}\end{array}$ \\
\hline Sex & & & & \\
$\quad$ Male & 16 & 20 & 6 & $42(57 \%)$ \\
$\quad$ Female & 15 & 9 & 7 & $31(43 \%)$ \\
Age (years) & & & & $3(4 \%)$ \\
$\quad \leq 1$ & 2 & 1 & 0 & $9(12 \%)$ \\
$1-5$ & 5 & 3 & 1 & $25(34 \%)$ \\
$6-10$ & 8 & 10 & 5 & $36(49 \%)$ \\
$11-14$ & 16 & 15 & & \\
\hline
\end{tabular}

All patients had fever at some point of their illness. The other common symptoms and signs at presentation are shown in Table 2. The clinical presentations included headache, vomiting, myalgia, diarrhea, and mucosal bleeding such as gum bleeding and epistaxis. Spontaneous bleeding was seen in 29 cases (39\%). It was more common during the first 5 days of illness. In 7 patients, the caretaker gave a history of fever with chills.

Hemoconcentration was detected in 42 patients with DHF and DSS on days 4 to 8 day of illness (mean 5.8 days). In 13 DSS patients, shock occurred on days 4 to 8 of their illness (mean 6 days). Five patients with DSS developed shock before the fever declined with the temperature at the time of shock ranged from 38.0 to 38.8 C. In 3 patients, fever declined 1-3 days after recovery from shock with no complications. The other 2 patients developed dyspnea after fluid resuscitation, and needed endotracheal intubation with mechanical ventilatory support, but eventually developed bacterial pneumonia. They were treated and recovered after treatment with antibiotics. Fever subsided on 6 and 12 days after shock, respectively.

Two patients ( 8 months and 2 years of age) developed convulsion on the first and fifth day of fever, both were diagnosed as having febrile convulsion, and were not considered as having unusual manifestation. Among the 17 patients who had underlying disease, all recovered well except for the patient who had hemoglobin $\mathrm{H} /$ hemoglobin Constant Spring with hemoglobin AEBart (described below). Of the 7 patients with a history of fever with chills, 6 patients recovered. The seventh patient had altered consciousness and liver failure as described below.

Unusual manifestations found in our patients are shown in Table 3. There were 9 patients with neurological manifestations; 6 with altered consciousness, and 3 with convulsions that could not be otherwise explained (Table 3). Among 6 patients with altered consciousness, 3 patients also had confusion and disorientation of time, place, and person, and 1 patient showed behavioral changes. Symptoms in 4 out of 6 patients occurred during the febrile phase or at the beginning of fever lysis (days 2, 4, 6, 8; body temperature 39.6, 37.8, 36.5, 37 C) in patients who were identified as DF, DHF, DF, and DSS, respectively. Lumbar puncture was performed in the first patient. The cerebrospinal fluid (CSF) revealed 8 white blood cells $/ \mathrm{mm}^{3}$ (no information on the differential count) with normal protein and sugar and open/close pressures of 30 and $20 \mathrm{~cm} \mathrm{H}_{2} \mathrm{O}$, respectively. Neurological symptoms in these 4 patients

Table 2. Clinical presentations of patients with DF, DHF and DSS ( $\mathrm{n}=73$ )

\begin{tabular}{lcccc}
\hline Clinical presentations & $\begin{array}{c}\text { DF } \\
(\mathbf{n = 3 1 )}\end{array}$ & $\begin{array}{c}\text { DHF } \\
(\mathbf{n = 2 9 )}\end{array}$ & $\begin{array}{c}\text { DSS } \\
(\mathbf{n}=\mathbf{1 3})\end{array}$ & $\begin{array}{c}\text { Total } \\
(\mathbf{n}=\mathbf{7 3})(\mathbf{\%})\end{array}$ \\
\hline Nausea/vomiting & 14 & 12 & 4 & $30(41 \%)$ \\
Mucosal bleeding & 10 & 12 & 7 & $29(40 \%)$ \\
Myalgia & 9 & 10 & 6 & $25(34 \%)$ \\
Headache & 12 & 7 & 3 & $22(30 \%)$ \\
Diarrhea & 10 & 8 & 3 & $21(29 \%)$ \\
& & & & \\
\hline
\end{tabular}


subsided spontaneously after 1 to 2 days following symptomatic treatment. Two patients came for followup 2 weeks after discharge and were well. The fifth patient was a 3-year-old girl who had a history of fever with chill. She developed altered consciousness on the third day of fever and acute liver failure. Computerized tomography (CT) of the brain revealed edema. She was diagnosed as having DHF with hepatic encephalopathy. She recovered with supportive treatment and was discharged well after 10 days following admission. At follow-up 2 weeks after discharge, she was well with normal liver function. The sixth patient with altered consciousness was a 12-year-old girl with thalassemia (hemoglobin $\mathrm{H} /$ hemoglobin Constant Spring with hemoglobin AEBart). She had fever with diarrhea for 2 days and then developed altered consciousness. She was treated at a local hospital for 2 days before transfer to our hospital. The laboratory findings on admission were compatible with fulminant hepatic failure and acute kidney injury. There was no evidence of plasma leakage. The patient deteriorated with disseminated intravascular coagulation and severe bleeding despite aggressive treatment including mechanical ventilatory support and hemodialysis. CT revealed multifocal hemorrhages in both cerebral hemispheres and impending transtentorial brain herniation. Her relatives requested to bring her home after 3 days of admission where she presumably died.
Convulsion was observed in 3 patients (Table 3 ). Two patients with DSS were resuscitated with a large amount of fluid including dextran and fresh frozen plasma and one patient also received massive blood transfusion because of massive upper gastrointestinal bleeding. One of these 2 DSS patients was referred to our hospital on the sixth day of her illness. She also had renal failure and multiple organ failure. She died on the third day of admission. Another patient with DHF developed brief episodes of drowsiness and convulsion on the second day of fever and was referred to our hospital on the seventh day of her illness. CT revealed generalized brain swelling. She recovered from drowsiness in 2 days and was discharged on the third day of admission. Two weeks after discharge, she was well and the neurological examination was normal.

Acute kidney injury and fulminant hepatic failure was observed in 1 patient (Table 3). A 4 year-old-girl developed acute kidney injury 2 days after fluid resuscitation for DSS with fulminant hepatic failure, hepatic encephalopathy, and respiratory distress because of massive pleural effusion, and required mechanical ventilatory support. She was referred to our hospital on the ninth day of her illness. She was treated with all available therapy including total body exchange transfusion (3 times) and hemodialysis (6 times). She recovered and was discharged after 36 days of hospitalization. At follow-up 2 weeks after discharge, she was well with normal kidney and renal function.

Table 3. Summary of unusual manifestations in patients with DF, DHF and DSS ( $n=73)$

\begin{tabular}{|c|c|c|c|c|}
\hline Unusual manifestations & $\begin{array}{c}\text { DF } \\
(n=31)\end{array}$ & $\begin{array}{c}\text { DHF } \\
(n=29)\end{array}$ & $\begin{array}{c}\text { DSS } \\
(n=13)\end{array}$ & $\begin{array}{c}\text { Total } \\
(n=73)(\%)\end{array}$ \\
\hline \multicolumn{5}{|l|}{ Altered consciousness (6) } \\
\hline Recovered in 2 days & 2 & 1 & 1 & $4(6)$ \\
\hline With fulminant hepatic failure & 0 & 1 & 0 & $1(1)$ \\
\hline With fulminant hepatic failure and kidney injury & $1^{\mathrm{a}}$ & 0 & 0 & $1(1)$ \\
\hline \multicolumn{5}{|l|}{ Convulsion (3) ${ }^{\mathrm{b}}$} \\
\hline Convulsion only & 0 & 0 & 2 & $2(3)$ \\
\hline With kidney injury & 0 & 1 & 0 & $1(1)$ \\
\hline Acute kidney injury and fulminant hepatic failure & 0 & 0 & 1 & 1(1) \\
\hline Congestive heart failure with fulminant hepatic & & & & \\
\hline failure and kidney injury & 1 & 0 & 0 & $1(1)$ \\
\hline IAHS & 0 & 1 & 0 & 1(1) \\
\hline Total & 4 & 4 & 4 & $12(16)$ \\
\hline
\end{tabular}

${ }^{\mathrm{a}}$ Underlying thalassemia, ${ }^{\mathrm{b}}$ after fluid resuscitation of shock in 2 patients, after massive blood transfusion in 1 patient, 'underlying ventricular septal defect 
Another unusual manifestation was observed in a 9-year-old boy with a ventricular septal defect who presented with a history of fever and rhinorrhea 1 week before admission. Three days before admission, he had a low grade fever and cough with increasing dyspnea. Physical examinations and laboratory findings were compatible with congestive heart failure and fulminant hepatic failure. Electrocardiography showed evidence of myocarditis with a right bundle branch block. The echocardiogram revealed a small size VSD with poor left ventricular function. The patient developed acute renal injury and cardiac arrest few hours after admission. He died 24 hours later. The dengue IgM test was positive.

Infection associated hemophagocytic syndrome (IAHS) was observed in a 10-month-old girl with DHF. IAHS was diagnosed by laboratory and bone marrow biopsy findings on the eighth day of fever. She was treated with one dose of intravenous immunoglobulin G. She recovered and was discharged after 10 days of hospitalization. Dengue IgM tested on the sixth day of fever was positive. She was well at follow-up one month after discharge with normal complete blood counts and liver function.

There were 9 patients who needed endotracheal intubation and mechanical ventilatory support because of pleural effusion and respiratory distress, pulmonary hemorrhage, and bacterial pneumonia. In hospital mortality was $2.7 \%$ (2/73). Including 1 patient who was brought back home in a moribund condition, overall, mortality was $4.1 \%$ (3/73).

\section{Discussion}

It must be noted that these patients are a selected population reported from a regional referral center and thus are not representative of a general dengue population. Among these 73 confirmed dengue patients, common manifestations were nausea, vomiting, myalgia, and headache, which are the usual symptoms associated with dengue infection. Diarrhea was present in $28.8 \%$ of DF, DHF, and DSS patients. Common unusual presentations were neurological symptoms, which occurred in 9 patients (12.3\%). Other manifestations were liver and kidney involvement. Diarrhea has not been recognized as a common clinical manifestation in dengue infection. In a study in Thailand from 1962 to 1964, diarrhea was recorded in only $6.4 \%$ of 78 dengue patients [15]. In an epidemiological study of dengue in school children at Ratchaburi, Thailand from 2006 to 2008, diarrhea was seen in $17.1 \%$ and $50.0 \%$ of DF and DHF patients, respectively [16]. A study of gastrointestinal manifestations in patients with dengue infection in the 2006 dengue epidemic in Mexico, diarrhea was found in $24 \%$ of 1,640 hospitalized patients [17]. In our study, we found diarrhea in $28.8 \%$. In 4 patients (4/21, $19.0 \%$ ) diarrhea was the main symptom that brought them to seek treatment.

Neurological manifestations have been increasingly reported in dengue infection $[3,5,8]$. Dengue encephalopathy and encephalitis may present in various forms including convulsions, pyramidal signs and behavioral abnormalities [18]. Neurological manifestations in dengue can be the result of direct virus invasion of the brain, capillary hemorrhage, disseminated intravascular coagulation, and metabolic abnormalities [19]. In a prospective study in Thailand from 1996 and 1998, 18.2\% (8/44) of the children with a preliminary diagnosis of viral encephalitis were proven to actually have dengue by serology and/or PCR of CSF. None of them had liver failure and their clinical course and prognosis were favorable [20]. In a study of patients with central nervous system dysfunction in southern Vietnam during 1995, 4.2\% (16/378) were found to have dengue infection. The most frequent neurological manifestations were impaired consciousness and convulsions [21]. In our study, altered consciousness was the most common neurological symptom. Four patients who developed encephalitis-like symptoms during the febrile stage or at the beginning of fever lysis without liver or kidney involvement, had a mild clinical course and all recovered completely. CSF findings in 1 patient were compatible with encephalitis. In 3 patients who developed convulsion, we could not ascertain whether the neurological manifestation was a consequence of dengue virus infection, fluid overload, complication, or other causes.

Intracranial hemorrhage is another cause of neurological manifestations in dengue, and it may be an important cause of persistent neurological abnormalities in survivors [18]. Most such reported cases were in patients with severe thrombocytopenia, prolonged shock, and coagulopathy as a result of fulminant hepatic failure as also described in our patient [6, 22-23].

In the present study, there was a patient with a case of IAHS who recovered after receiving intravenous immunoglobulin. Although hemophagocytosis is an uncommon manifestation of dengue, a 
few cases have been reported in patients with DHF [24-26]. IAHS should be suspected in dengue patients who have persistent fever and cytopenia following resolution of the plasma leakage (critical) phase of DHF [26].

In this study, one patient with congenital heart disease developed congestive heart failure with evidence of myocarditis and multiple organ failure, representing a rare manifestation of dengue that has been previously reported in a 13-year-old Thai patient [27].

In our patients, acute renal injury occurred after prolonged shock and respiratory failure that required endotracheal intubation, fluid resuscitation, massive pleural effusion, and/or bacterial pneumonia. Acute fulminant hepatic failure occurred in the febrile phase of illness and after prolonged shock. Exchange transfusion and hemodialysis may be helpful in lowering mortality in patients with prolonged shock complicated by multiple organ failure and metabolic abnormalities. The most important management of dengue to prevent shock is careful fluid management before and during the transition from the febrile phase to the plasma leakage stage.

This study has several limitations. The data were collected retrospectively. The long-term outcome of patients with unusual manifestations or complications who did not come for follow-up could not be ascertained. We did not perform dengue virus detection in the CSF in patients with neurological manifestations. Our patients were seen at a pediatric referral center and results might be different from those seen in smaller centers. However, this study described neurological manifestations in relation to the phase of dengue illness and found that all patients with altered consciousness in the febrile phase of dengue infection recovered.

\section{Conclusion}

Altered consciousness was the most common neurological manifestation that occurred in both DF and DHF (including DSS) patients. Liver, renal, and respiratory involvement were common complications of prolonged shock; emphasizing the need for careful monitoring and extreme caution with fluid replacement in patients with DHF and DSS.

\section{Acknowledgements}

The authors thank the nursing and medical staff who cared for these children. This study was supported by funds from the Faculty of Medicine, Khon Kaen University. We thank Prof. Dr. Yukifumi Nawa, Research Affairs, Faculty of Medicine, Khon Kaen University for his help. The authors have no conflicts of interest to declare.

\section{References}

1. World Health Organization. Dengue haemorrhagic fever: diagnosis, treatment, prevention and control, 2nd ed. Geneva: WHO; 1997.

2. Nimmanitya S, Thisyakorn U, Hemsrichart V. Dengue hemorrhagic fever with unusual manifestations. Southeast Asian J Trop Med Public Health. 1987; 18: 398-406.

3. Pancharoen C, Thisyakorn U. Neurological manifestations in dengue patients. Southeast Asian J Trop Med Public Health. 2001; 32:341-5.

4. Sirivichayakul C, Sabcharoen A, Chanthavanich P, Pengsaa K, Chokejindachi W, Prarinyanupharb V. Dengue infection with unusual manifestation; a case report. J Med Assoc Thai. 2000; 83:325-9.

5. Janssen HLA, Bienfait HP, van Duinen SG, Vriesendorp $\underline{\mathrm{R}, \text { Schimsheimer RJ, et al. Fatal cerebral edema }}$ associated with primary dengue infection. J Infect. 1998; 36:344-6.

6. Kamath SR, Ranjit S. Clinical features, complications and atypical manifestations of children with severe forms of dengue hemorrhagic fever in South India. Indian J Pediatr. 2006; 73:889-95.

7. Jhamb R, Kumar A, Ranga GS, Rathi N. Unusual manifestations in dengue outbreak 2009, Delhi, India. J Commun Dis. 2010; 42:255-61.

8. Verma R, Sharma P, Garg RK, Atam V, Singh MK, Mehrotra HS. Neurological complications of dengue fever: Experience from a tertiary center of north India. Ann Indian Acad Neurol. 2011; 14:272-8.

9. Chongsrisawat V, Hutagalung Y, Poovorawan Y. Liver function test results and outcomes in children with acute liver failure due to dengue infection. Southeast Asian J Trop Med Public Health. 2009; 40: 47-53.

10. Laoprasopwattana K, Pruekprasert P, Dissaneewate P. Outcome of dengue hemorrhagic fever-caused acute kidney injury in Thai children. J Pediatr. 2010; 157:303-9.

11. World Health Organization Regional Office for SouthEast Asia. Comprehensive guidelines for prevention and control of dengue and dengue haemorrhagic fever: revised and expanded edition. New Delhi; World Health Organization Regional Office for South- 
East Asia; 2011.

12. Lapphra K, Sangcharaswichai A, Chokephaibulkit K, Tiengrim S, Piriyakarnsakul W, Chakorn T, et al. Evaluation of an NS1 antigen detection for diagnosis of acute dengue infection in patients with acute febrile illness. Diagn Microbiol Infect Dis. 2008; 60:387-91.

13. Suchy FJ. Fulminant hepatic failure. In: Kliegman RM, Stanton BF, St. GemeIII JW, Schor NF, Behrman RE, editors: Nelson's Textbook of Pediatrics, 19th ed. Philadelphia: WB Saunders; 2011. p. 1412-5.

14. Mehta RL, Kellum JA, Shah SV, Molitoris BA, Ronco C, Warnock DG, et al. Acute kidney injury Network: report of an initiative to improve outcomes in acute kidney injury. Crit Care. 2007; 11:R31

15. Nimmannitya S, Halstead SB, Cohen SN, Margiotta MR. Dengue and chikungunya virus infection in man in Thailand, 1962-1964. Observations on hospitalized patients with hemorrhagic fever. Am J Trop Med Hyg. 1969; 18:954-71.

16. Sirivichayakul C, Limkittikul K, Chanthavanich P, Jiwariyavej V, Chokejindachai W, Pengsaa K, et al. Dengue infection in children in Ratchaburi, Thailand: a cohort study. II. Clinical manifestations. PLoS Negl Trop Dis. 2012; 6: e1520.

17. Ramos-De La Medina A, Remes-Troche JM, GonzalezMedina MF, Anitua-Valdovinos Mdel M, Ceron T, Zamudio C, et al. Abdominal and gastrointestinal symptoms of dengue fever. Analysis of a cohort of 8559 patients. Gastroenterol Hepatol. 2011; 34:243-7.

18. Gulati S, Maheshwari A. A typical manifestations of dengue. Trop Med Int Health. 2007; 12:1087-95.

19. Lum LC, Lam SK, Choy YS, George R, Harun F.
Dengue encephalitis: a true entity? Am J Trop Med Hyg. 1996; 54:256-9.

20. Kankirawatana P, Chokephaibulkit K, Puthavathana P, Yoksan S, Apintanapong S, Pongthapisit V. Dengue infection presenting with central nervous system manifestation. J Child Neurol. 2000; 15:544-7.

21. Solomon T, Dung NM, Vaughn DW, Kneen R, Thao LT, Raengsakulrach B, et al. Neurological manifestations of dengue infection. Lancet. 2000; 355: 1053-9.

22. de Souza LJ, Martins AL, Paravidini PC, Nogueira RM, Gicovate Neto C, Bastos DA, et al. Hemorrhagic encephalopathy in dengue shock syndrome: a case report. Braz J Infect Dis. 2005; 9:257-61.

23. Bunnag T, Kalayanarooj S. Dengue shock syndrome at the emergency room of Queen Sirikit National Institute of Child Health, Bangkok, Thailand. J Med Assoc Thai. 2011; 94Suppl 3:S57-63.

24. Veerakul G, Sanpakit K, Tanphaichitr VS, Mahasandana C, Jirarattanasopa N. Secondary hemophagocytic lymphohistiocytosis in children: an analysis of etiology and outcome. J Med Assoc Thai. 2002; 85: S530-41.

25. Jain D, Singh T. Dengue virus related hemophagocytosis: a rare case report. Hematology. 2008; 13:286-8.

26. Tan LH, Lum LC, Omar SF, Kan FK. Hemophagocytosis in dengue: comprehensive report of six cases. J Clin Virol. 2012; 55:79-82.

27. Promphan W, Sopontammarak S, Pruekprasert P, Kajornwattanakul W, Kongpattanayothin A. Dengue myocarditis. Southeast Asian J Trop Med Public Health. 2004; 35:611-3. 